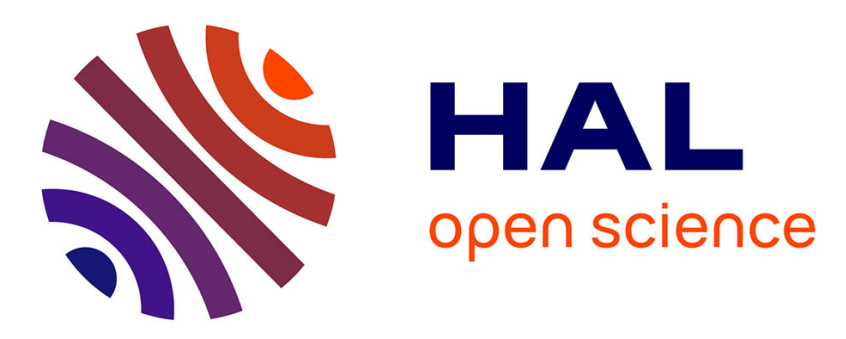

\title{
Metric for the measurement of the quality of complex beams. Theoretical study
}

S. Kaim, Julien Lumeau, Vadim Smirnov, B. Ya. Zel'Dovich, Leonid B. Glebov

\section{- To cite this version:}

S. Kaim, Julien Lumeau, Vadim Smirnov, B. Ya. Zel'Dovich, Leonid B. Glebov. Metric for the measurement of the quality of complex beams. Theoretical study. Journal of the Optical Society of America. A, Optics and image science, 2015, 32 (4), pp.538-548. hal-01274395

\section{HAL Id: hal-01274395 \\ https://hal.science/hal-01274395}

Submitted on 13 Mar 2019

HAL is a multi-disciplinary open access archive for the deposit and dissemination of scientific research documents, whether they are published or not. The documents may come from teaching and research institutions in France or abroad, or from public or private research centers.
L'archive ouverte pluridisciplinaire HAL, est destinée au dépôt et à la diffusion de documents scientifiques de niveau recherche, publiés ou non, émanant des établissements d'enseignement et de recherche français ou étrangers, des laboratoires publics ou privés. 


\title{
Metric for the Measurement of the Quality of Complex Beams. Theoretical Study
}

\author{
Sergiy Kaim, ${ }^{1}$ Julien Lumeau, ${ }^{2}$ Vadim Smirnov, ${ }^{3}$ Boris Zeldovich,,${ }^{1, *}$ Leonid Glebov, \\ ${ }^{I}$ CREOL, The College of Optics and Photonics, University of Central Florida, Orlando, FL 32816 \\ ${ }^{2}$ Aix-Marseille Université, CNRS, Centrale Marseille, Institut Fresnel, UMR 7249, 13013 Marseille, France \\ ${ }^{3}$ OptiGrate Corp 562 South Econ Circle, Oviedo, Florida 32765-4311 \\ *Corresponding author: boris@creol.ucf.edu
}

Received Month X, XXXX; revised Month X, XXXX; accepted Month X, XXXX; posted Month X, XXXX (Doc. ID XXXXX); published Month X, XXXX

\begin{abstract}
We study theoretically various definitions of laser beam width in a given cross-section. Quality of the beam is characterized by dimensionless Beam Propagation Products BPPs $\Delta x \cdot \Delta \theta_{x} / \lambda$, which are different for 21 definitions, but are close to 1. Six particular beams are studied. In the process we had to review the properties of Fourier transform of various modifications: Physical Fourier Transform (PFT), Mathematical Fourier Transform (MFT), Discrete Fourier Transform (DFT), and relationships between them. We found axially-symmetric self-MFT function, which may be useful for description of diffraction-quality beams. In Appendices we illustrate the thesis "Fourier lives on the singularities of original".

OCIS codes: (120.4800) Optical standards and testing, (070.2465) Finite analogs of Fourier transforms,

(070.7345) Wave propagation
\end{abstract}

\section{Introduction}

Problem of characterizing and measuring transverse quality of a laser beam has long history of studies. It is worth mentioning specifically monograph [1] and papers, e.g. [2]. Most laser beams have very small angular divergence $\theta_{x} \quad\left(\leq 10^{-2} \mathrm{rad}\right)$. Transformation of such beams by lenses without aberrations may separately change $\delta \theta_{x}$ and the waist radius $\Delta x$. However, the product $\Delta x \cdot \Delta \theta_{x}$ (of dimension meters) is not changed by such transformation, and for almost-diffraction-quality beams is of the order of wavelength $\lambda$. Particular dimensionless quantity $\Delta x \cdot \Delta \theta_{x} / \lambda$ depends on the formal definition of $\Delta x$ and $\Delta \theta_{x}$ in theoretical discussions of the problem, and depends on the measuring procedures in experiment.

One possible definition of $\Delta x$ and $\Delta \theta_{x}$ is root-mean-square and related to it dimensionless parameter $M_{x}{ }^{2}$, adopted as ISO standard [3]:

$$
\begin{gathered}
\Delta x_{r m s}=\sqrt{\left\langle(x-\langle x\rangle)^{2}\right\rangle}, \quad \Delta \theta_{r m s}=\sqrt{\left\langle\left(\theta_{x}-\left\langle\theta_{x}\right\rangle\right)^{2}\right\rangle}, \\
M_{x}{ }^{2}=(4 \pi / \lambda) \cdot \Delta x_{r m s} \cdot \Delta \theta_{r m s}
\end{gathered}
$$

Separate measurement of $M_{x}{ }^{2}$ and $M_{y}{ }^{2}$ is often necessitated by not quite axially-symmetric character of the beam, including possible astigmatism. Particular coefficient, $4 \pi$, is chosen in such a manner, that minimum value of $M_{x}{ }^{2}$ equals 1 , and is achieved for ideal beam with perfect Gaussian profile.

It is assumed in (1) that $\Delta x_{\mathrm{rms}}$ is measured at the $z$-position of its minimum (in focal waist in the case of focused beam), while $\Delta \theta_{\mathrm{rms}}$ is measured in the far field zone of the beam. Quite often in experiment the far-field zone with its angular distribution of intensity $I\left(\theta_{x}\right)$ is substituted by the profile $I_{\text {waist }}\left(x=F \cdot \theta_{x}\right)$ in the focal plane of a positive lens with focal distance $F$. This often leads to confusion, which parameter, $\Delta x_{\mathrm{rms}}$ or $\Delta \theta_{\mathrm{rms}}$ corresponds to near-field, and which one is related to the far-field. Luckily, this modest confusion in terminology does not result in the change of $M_{x}{ }^{2}$, because as we have already mentioned, the product $\Delta x \cdot \Delta \theta_{x}$ is invariant under transformation by paraxial optical elements without aberrations.

Many researchers have noted that the quantities $\Delta x_{\mathrm{rms}}, \Delta \theta_{\mathrm{rms}}$ and therefore $M_{x}{ }^{2}=4 \pi \cdot \Delta x_{\mathrm{rms}} \cdot \Delta \theta_{\mathrm{rms}} / \lambda$ put too much emphasis upon distant wings of distributions $I_{0}(x)$ and $I_{1}\left(\theta_{x}\right)$, e.g. [4-7]. This includes an experimental paper by Lantigua et al., [7].

Our personal preference is the use of criterion "width of the slit, containing $85 \%$ of total power", and ratio of Beam Propagation Product to the BPP for ideal Gaussian beam by the same criterion. The chosen fraction $85 \%$, seems to be reasonable for energy-delivering applications of laser beams. Meanwhile the slit technology is relatively easy in implementation of field devices.

Given field $E_{0}(x, y)$ in the near-field zone, one finds intensity profile there $I_{0}(x, y)=\left|E_{0}(x, y)\right|^{2}$. Besides that, angular amplitude profile, i.e. amplitude profile in the far-field zone is proportional to

$$
G_{2}\left(\theta_{x}, \theta_{y}\right)=\frac{1}{2 \pi} \iint E_{0}(x, y) e^{-i k\left(x \cdot \theta_{x}+y \cdot \theta_{y}\right)} d x d y .
$$

The resultant angular intensity profile is $I_{2}\left(\theta_{x}, \theta_{y}\right)=$ $\left|G_{2}\left(\theta_{x}, \theta_{y}\right)\right|^{2} \cdot \operatorname{In}(1 \mathrm{D}+z)$ case $E_{0}(x, y) \equiv E_{0}(x)$, and

$$
G_{1}\left(\theta_{x}\right)=\frac{1}{\sqrt{2 \pi}} \int E_{0}(x) \exp \left(-i k x \cdot \theta_{x}\right) d x
$$

and $I_{1}\left(\theta_{x}\right)=\left|G_{1}\left(\theta_{x}\right)\right|^{2}$. Here and below wave number $k=2 \pi / \lambda$, and $\lambda$ is wavelength in the medium of propagation path (typically in vacuum).

For that reason in Section 2 we review three definitions of Fourier Transform (FT): Physical (PFT), Mathematical (MFT) and Discrete (DFT). In Section 2B we discuss 1-dimensional self- 
MFT functions. In Section $2 \mathrm{C}$ we discuss DFT and its relationship to $\mathrm{PFT}$ and MFT. In Section $2 \mathrm{D}$ we introduce new axially-symmetric self-MFT function, based on 1D self MFT function $1 / \cosh (x \sqrt{\pi / 2})$.

In Section 3 we consider 21 quantitatively different definitions of the beam width, and calculate a table of those width for 6 different smooth transverse profiles in the near field. We calculate also their far-field profiles. In this manner we were able to find Beam Propagation Products (BPP) for these beams according to the 21 different criteria. The tables of BPP are compiled in assumption that one and the same criterion of width (out of 21 considered) is taken both for near-field and for far-field. Meanwhile we provide the data of calculations, which allow to take one criterion in near-field and another in the far-field, and thus arrange for such a compound BPP. Section 4 summarizes the results of the work. In Appendix A we illustrate the thesis "Fourier transform lives on the singularities of original". While this thesis is not scientifically new, we failed to find a mathematical textbook or a paper with its presentation. In Appendix B we apply DFT for the study of self-MFT functions.

\section{Fourier Transforms (FT): Physical (PFT), Mathematical (MFT), Discrete (DFT). Self-MFT functions}

\section{A. Physical Fourier Transform (PFT)}

We start with Fourier Transform (FT) as it is used in Physics (PFT). Consider the function $f(x)$ of real variable $x$ (for example, of dimensions of Cartesian coordinate, $[x]=[$ meter $]$ ). This function may have real or complex values. We define new function $G(q)$ of new real argument $q$ (of dimensions $[q]=$ [radian/meter]) by

$$
G(q)=\frac{A}{\sqrt{2 \pi}} \int_{-\infty}^{+\infty} \exp (i q x) f(x) d x
$$

where $A \neq 0$ is some constant. Then, as it is well known in mathematics, under certain (not very restrictive) conditions, original function $f(x)$ may be found by inverse Fourier transformation,

$$
f(x)=\frac{1}{A \sqrt{2 \pi}} \int_{-\infty}^{+\infty} \exp (-i q x) G(q) d q .
$$

Traditional choices of constant $A$ are, for example $=1$, $A=\sqrt{2 \pi}, A=1 / \sqrt{2 \pi}$, but any $A \neq 0$, even a complex number, does the job. Formula (4) defines linear operator of Physical FT; its maps space of functions $f(x)$ of argument $x$ onto the space of functions $G(q)$ of a different argument $q$, dimensions of $q$ being inverse to the dimensions of $x:[q]=[1 / x]$. Parseval's theorem claims that

$$
|A|^{2} \cdot \int_{-\infty}^{+\infty}|f(x)|^{2} d x=\int_{-\infty}^{+\infty}|G(q)|^{2} d q .
$$

It looks especially elegant for $A=1$.

\section{B. Mathematical Fourier Transform (MFT)}

If one wants to discuss eigenfunctions of FT, then FT operator must map space functions $f(y)$ onto itself, $G(y)$. In that case dimensions $[q \equiv y]$ coincides with dimensions $[1 / y]$. In other words, argument $x$ of functions $f(x)$ for Mathematical FT (MFT) should be dimensionless. All this gives the justification to the following definition of MFT operator as

$$
\operatorname{MFT}\{f\}(x)=h(x)=\frac{1}{\sqrt{2 \pi}} \int_{-\infty}^{+\infty} \exp \left(i x x^{\prime}\right) f\left(x^{\prime}\right) d x^{\prime} .
$$

Parseval's theorem shows that MFT operator is unitary:

$$
\int|h(x)|^{2} d x=\int\left|f\left(x^{\prime}\right)\right|^{2} d x^{\prime}
$$

Inverse PFT (5) differs (at $A=1$ ) from original PFT (4) only by the sign of phase in the exponential. It allows to conclude that application of MFT operator to a function $f(x)$ two times returns $f(-x)$ :

$$
(M F T)^{2}\{f\}(x)=f(-x) .
$$

From that one gets

$$
(M F T)^{4}\{f\}(x)=f(x), \quad \text { or }(M F T)^{4}=\hat{1},
$$

i.e. 4-th power of MFT operator is unit operator. As a result, eigenvalues $\Lambda$ of MFT operator satisfy condition $\Lambda^{4}=1$,

$$
\operatorname{MFT}\{h\}(x)=\Lambda \cdot h(x), \Lambda^{4}=1 .
$$

Thus there are only 4 possible eigenvalues of MFT: $\Lambda_{0}=1$, $\Lambda_{1}=i, \Lambda_{2}=-1, \Lambda_{3}=-i$ (or $\left.\Lambda_{n}=i^{n}, n=0,1,2,3\right)$.

Differentiation and integration by parts in MFT Eq. (7) allows to show that if $f(x)$ is an eigenfunction of MFT with eigenvalue $\Lambda_{f}$, i.e. if

then functions

$$
\operatorname{MFT}\{f\}(x)=\Lambda_{f} \cdot f(x)
$$

$$
g(x)=\left(x-\frac{d}{d x}\right) f(x), \quad h(x)=\left(x+\frac{d}{d x}\right) f(x)
$$

are also eigenfunctions of MFT, and

$$
\Lambda_{g}=i \cdot \Lambda_{f}, \quad \Lambda_{h}=-i \cdot \Lambda_{f} .
$$

Function $g_{0}(x)=\exp \left(-x^{2} / 2\right)$ is a well-known eigenfunction of MFT, with eigenvalue $\Lambda_{0}=+1$. Moreover, Hermite polynomials $H_{n}(x)$ multiplied by $g_{0}(x)$, i.e.

$$
H_{n}(x) \exp \left(-x^{2} / 2\right) \text {, }
$$

up to constant factors, can be produced from $g_{0}(x)$ by application of "creation operator" $(x-d / d x)$ sequentially $n$ times. Therefore they are eigenfunctions of MFT, with eigenvalues $\Lambda_{n}=i^{n}$.

Another function,

$$
c_{0}(x)=[\cosh (x \sqrt{\pi / 2})]^{-1},
$$

is also an eigenfunction of MFT, with eigenvalue $\Lambda_{0}=+1$. Main difference between $g_{0}(x)$ and $c_{0}(x)$ is in their asymptotic behavior at $|x| \rightarrow \infty: g_{0}(x)=\exp \left(-x^{2} / 2\right)$ (exact); meanwhile $c_{0}(x) \doteq 2 \cdot \exp (-|x| \sqrt{\pi / 2})$. Functions const $g_{g} \cdot g_{0}(x)$ and const $_{c} \cdot c_{0}(x)$, normalized to $\int|f(x)|^{2} d x=1$, have almost $100 \%$ overlapping integral:

$$
\left\|g_{0} c_{0}\right\|^{2} \equiv \frac{\left[\int_{-\infty}^{+\infty} g_{0}(x) c_{0}(x) d x\right]^{2}}{\left[\int_{-\infty}^{+\infty}\left|g_{0}(y)\right|^{2} d y\right] \cdot\left[\int_{-\infty}^{+\infty}\left|c_{0}(z)\right|^{2} d z\right]}=0.994
$$

Property $(\mathrm{MFT})^{4}=\hat{1}$ allows to construct eigenfunctions of MFT out of an arbitrary function $f(x)$ of dimensionless argument. For example,

$$
f_{\beta}(x)=f(x)+i^{\beta} M F T\{f\}(x)+i^{2 \beta}(M F T)^{2}\{f\}(x)+
$$




$$
+i^{3 \beta}(M F T)^{3}\{f\}(x)
$$

is an eigenfunction of MFT with eigenvalue $\Lambda=i^{\beta}$, where $\beta$ is any integer number from 0 to 3 . For the case with $\Lambda=+1$, i.e. when $\beta=0$ is considered, formula of the type Eq. (18) was suggested in $[8,9]$.

Curious examples of MFT eigenfunctions are

$$
r_{\text {even }}(x)=\frac{1}{\sqrt{|x|}}, \quad r_{\text {odd }}(x)=\frac{x}{|x|} \cdot \frac{1}{\sqrt{|x|}},
$$

with respective eigenvalues $\Lambda_{\text {even }}=+1, \Lambda_{\text {odd }}=+i$. However, each of them has logarithmically divergent normalization integral (both at $|x| \rightarrow 0$ and at $|x| \rightarrow \infty$ ).

\section{C. Discrete Fourier Transform (DFT) properly. Approximation of PFT by DFT.}

Discrete Fourier Transform is usually introduced as an approximation for Physical FT. Consider function $f(x)$ at the interval $a \leq x<a+L$, and for definiteness let dimensions of $x$ be $[x]=[$ meters $]$. Let us characterize this function by its values at the set of $N$ equidistant points $n=0,1, \ldots, N-1$,

$$
\begin{gathered}
x_{0}=a, x_{1}=a+s_{x}, \ldots, x_{n}=a+n s_{x}, \ldots, \\
x_{N-1}=a+(N-1) s_{x} ; s_{x}=L / N .
\end{gathered}
$$

Here $s_{x}$ is step of $x$-coordinate. It is convenient to assume that function $f(x)$ is continued outside the interval $a \leq x<a+L$ in a periodic manner with period $L$, so that $f(x)=f(x+L)$. Then one can consider extra point $x_{\text {extra }}=a+s_{x} N \equiv a+L$ with the value $f(a+L)=f(a) \equiv f_{0}$, which is already accounted for by $f_{0}$. Corresponding vector $\vec{f}$ of $N$-dimensional linear space has components

$$
\vec{f}=\left[f_{0}=f\left(x_{0}\right), f_{1}=f\left(x_{1}\right), \ldots, f_{N-1}=f\left(x_{N-1}\right)\right] .
$$

Function $G(q)$ (i.e. PFT from (4)) may be approximated by trapezoid formula

$$
\begin{gathered}
G(q) \approx \frac{A}{\sqrt{2 \pi}} S_{x}\left[0.5 f_{0} e^{i q x_{0}}+f_{1} e^{i q x_{1}}+f_{2} e^{i q x_{2}}+\cdots+\right. \\
\left.+f_{N-1} e^{i q x_{N-1}}+0.5 f\left(x_{\text {extra }}\right) e^{i q x_{e x t r a}}\right]
\end{gathered}
$$

Periodicity assumption yields $f\left(x_{\text {extra }}\right) \equiv f_{0}$. Evidently, there are only $N$ linear independent values of function $G(q)$ defined by (22). To express this idea, we can choose to consider $N$ discrete values of argument $q$ :

$$
q_{0}=0, q_{1}=s_{q}, q_{2}=2 s_{q}, \ldots, q_{N-1}=(N-1) s_{q} .
$$

Periodicity condition in $x$-coordinate with period $L$ may be satisfied, if the value of step $s_{q}$ in $q$-space is chosen as $s_{q}=2 \pi / L$ (of dimensions [radian/meter]). In that case $f\left(x_{\text {extra }}\right) e^{i q_{m} \cdot x_{\text {extra }}}=$ $=f_{0} e^{i q_{m} \cdot x_{0}}$, and trapezoid approximation for $G(q)$ becomes

$$
\begin{gathered}
G_{m} \equiv G\left(m \cdot s_{q}\right) \equiv G\left(m \cdot \frac{2 \pi}{L}\right) \approx \\
\approx \frac{L \cdot A}{N \sqrt{2 \pi}} \exp \left(2 \pi i \frac{m a}{L}\right) \sum_{n=0}^{N-1} f_{n} \exp \left(2 \pi i \frac{n \cdot m}{N}\right) .
\end{gathered}
$$

Vector $\vec{g}$ of $N$-dimensional linear space is called Discrete Fourier Transform (DFT) of vector $\vec{f}$ from the same space, if its components are defined by

$$
g_{m}=(D F T\{f\})_{m}=\frac{1}{\sqrt{N}} \sum_{n=0}^{N-1} f_{n} \exp \left(2 \pi i \frac{n \cdot m}{N}\right) .
$$

Operator of DFT is implemented in every widely used mathematical software package like Mathcad, MatLab, Maple, Mathematica etc. What we were able to formulate here is that Physical FT G (q) from Eq. (4) may be approximated by

$$
G\left(q_{m} \equiv \frac{2 \pi m}{L}\right) \approx \exp \left(2 \pi i \frac{m a}{L}\right) \frac{L \cdot A}{\sqrt{2 \pi N}}(D F T\{f\})_{m} .
$$

Intuitively it is clear that Discrete FT Eq. (25) is a certain approximation of Physical FT. What is important is the particular $q_{m}$-dependent coefficient in Eq. (26) expressing PFT via DFT.

Remarkable mathematical facts about operator DFT defined by (25), are the following: 1) DFT is unitary operator in $\mathrm{N}^{-}$ dimensional linear space; 2) Inverse DFT operator (IDFT) looks also as approximation of Eq. (5) of inverse PFT, but is actually exact inverse operator with respect to DFT:

$$
f_{n}=(\operatorname{IDFT}\{\vec{g}\})_{n}=\frac{1}{\sqrt{N}} \sum_{m=0}^{N-1} g_{m} \exp \left(-2 \pi i \frac{n \cdot m}{N}\right) .
$$

The proof of this fact uses formula for the sum of geometrical progression:

$$
\sum_{k=0}^{N-1} \eta^{k}=\left[N, \text { if } \eta=1 ;\left(1-\eta^{N}\right) /(1-\eta) \text { otherwise }\right],
$$

with account of $\eta=\exp [2 \pi i(n-m) / N]$.

Additional problem to be covered is that physically both positive and negative values of $q[\mathrm{rad} /$ meter $]$ in (4), (5) are important. At first glance, $q_{m}=s_{q} \cdot m=2 \pi m / L$ with $m=$ $=0,1, \ldots, N-1$, cover positive values of $q$ only. This difficulty is resolved rather simply. For values $q_{N / 2}=N \pi / L$ exponential factors $\exp \left(i q_{m} \cdot x_{n}\right)=\exp \left(i q_{m} \cdot a\right) \cdot \exp (i \pi n)$ oscillate versus $n$ as $\exp (i \pi n) \equiv(-1)^{n}$. This is a manifestation of the failure of discretization of $f(x)$ into $f_{n}=f\left(x_{n}\right)$. In other words, we expect PFT of our function $f(x)$ to be negligibly small at $q_{m}$ with $m \approx N / 2$. On the other hand, subtracting $Q=2 \pi N / L$ from any of $q_{m}$ does not change exponential factors in DFT. Indeed, $\exp \left[i\left(q_{m}-Q\right) x_{n}\right]=\exp \left[i\left(q_{m}-Q\right) a\right] \cdot \exp \left[i q_{m} s_{x} n\right]$.

$\cdot \exp \left[-i Q s_{x} n\right]$. But $\exp \left[-i Q n s_{x}\right]=\exp (-2 \pi i n) \equiv 1$. Therefore one can subtract $Q=2 \pi N / L$ from any $q_{m}$ without changing the resultant DFT. We can introduce function

$$
\operatorname{phys}(m)=\left\{\begin{array}{r}
m, \quad \text { if } m<N / 2 \\
-N+m, \quad \text { otherwise }
\end{array},\right.
$$

so that $q_{m}$ (physical) $=s_{q} \cdot \operatorname{phys}(m)$ represent positive $q$ in the range $0 \leq m<N / 2$ and negative $q=-Q+q_{m}$ in the range $N / 2 \leq m \leq N-1$.

\section{D. Generation of eigenfunctions of 2D MFT (Mathematical Fourier Transform) via eigenfunctions of 1D MFT.}

The definition of Mathematical Fourier Transform for functions of two dimensionless variables $x, y$ is a trivial generalization of $1 \mathrm{D}$ case

$$
2 D \operatorname{MFT}\{f\}(x, y)=\frac{1}{2 \pi} \int_{-\infty}^{\infty} \int^{i\left(x x^{\prime}+y y^{\prime}\right)} f\left(x^{\prime}, y^{\prime}\right) d x^{\prime} d y^{\prime} .
$$


Consider two eigenfunctions of 1D MFT: $f_{1}(x)$ and $f_{2}(x)$, with eigenvalues $\Lambda_{1}=(i)^{\beta_{1}}$ and $\Lambda_{2}=(i)^{\beta_{2}}$, respectively. Functions $f_{1}(x)$ and $f_{2}(x)$ may be identical; in that case $\Lambda_{1} \equiv \Lambda_{2}$. Besides that $f_{1}(x)$ and $f_{2}(x)$ may be different eigenfunctions of MFT with the same or with different eigenvalues $\Lambda_{1}$ and $\Lambda_{2}$. In any of these cases factorized function of two dimensionless variables

$$
F_{\text {factor }}(x, y)=f_{1}(x) f_{2}(y), \Lambda_{\text {factor }}=\Lambda_{1} \cdot \Lambda_{2}
$$

is an eigenfunction of unitary 2D MFT operator (30), and $\Lambda_{\text {factor }}=\Lambda_{1} \cdot \Lambda_{2}$. The proof of this simple statement is based on factorization of exponential kernel in 2D MFT (30):

$$
e^{i x \cdot x^{\prime}+i y \cdot y^{\prime}}=e^{i x \cdot x^{\prime}} \cdot e^{i y \cdot y^{\prime}} .
$$

Besides that the scalar product $\left(\mathbf{r} \cdot \mathbf{r}^{\prime}\right) \equiv x x^{\prime}+y y^{\prime}$ in that kernel is invariant with respect to simultaneous rotation of coordinates by arbitrary angle $\psi$ :

$$
\begin{gathered}
(x, y)_{\text {new }}=(x, y) \hat{R}, \quad\left(x^{\prime}, y^{\prime}\right)_{\text {new }}=\left(x^{\prime}, y^{\prime}\right) \hat{R} \\
\hat{R}=\left(\begin{array}{cc}
\cos \psi & \sin \psi \\
-\sin \psi & \cos \psi
\end{array}\right) .
\end{gathered}
$$

Therefore another function,

$$
\begin{gathered}
F_{n e w, \psi}(x, y)=f_{1}\left(x_{n e w}\right) f_{2}\left(y_{n e w}\right)= \\
=f_{1}(x \cos \psi+y \sin \psi) \cdot f_{2}(-x \sin \psi+y \cos \psi),
\end{gathered}
$$

which generally is not factorized into $h_{1}(x) \cdot h_{2}(y)$, is still an eigenfunction of 2D MFT with $\Lambda_{\text {new }}=\Lambda_{1} \cdot \Lambda_{2}$. Linearity of $2 \mathrm{D}$ MFT operator guarantees that any superposition of such functions with $\psi$-dependent weight $W(\psi)$,

$$
F_{\text {superp }}(x, y)=
$$

is still an eigenfunction of $2 \mathrm{D}$ MFT. Using polar coordinates $x=\rho \cos \varphi, y=\rho \sin \varphi$ in $x, y$-plane, one can transform this superposition to

$$
\begin{gathered}
F_{\text {superp }}(x, y)=F_{\text {new }}(\rho, \varphi)= \\
=\int_{0}^{2 \pi} W(\psi) f_{1}(\rho \cos (\varphi-\psi)) f_{2}(\rho \sin (\varphi-\psi)) d \psi
\end{gathered}
$$

Let us assume that function $W(\psi)$ is periodic with period $2 \pi$, i.e. $W(\psi+2 \pi)=W(\psi)$. Introducing new variable $\alpha=\varphi-\psi$, one transforms integral (36) up to a factor $(-1)$ into

$$
F_{n e w}(\rho, \varphi)=-\int_{0}^{2 \pi} W(\varphi+\alpha) \cdot f_{1}(\rho \cos \alpha) \cdot f_{2}(\rho \sin \alpha) d \alpha
$$

A minor problem may arise, if the integral in the right-handside of Eq. (37) turns out, for some or other symmetry reason, to be exactly zero. Then one gets function equal to zero identically, which is not interesting, albeit may formally be considered as an eigenfunction of any linear operator.

Special interest is presented by the case when $W(\psi)=$ $=(-1 / 2 \pi) \exp (i m \psi)$, to elucidate the rotation symmetry in $(x, y)$-plane. Then

$$
\begin{gathered}
F_{n e w}(\rho, \varphi)=e^{i m \varphi} R_{m}(\rho) \\
R_{m}(\rho)=\frac{1}{2 \pi} \int_{0}^{2 \pi} e^{i m \alpha} f_{1}(\rho \cos \alpha) f_{2}(\rho \sin \alpha) d \alpha .
\end{gathered}
$$

We are especially interested in the case of completely axiallysymmetric $(m=0)$ self $2 \mathrm{D}$ MFT functions

$$
R_{0}(\rho)=\frac{1}{2 \pi} \int_{0}^{2 \pi} f_{1}(\rho \cos \alpha) f_{2}(\rho \sin \alpha) d \alpha
$$

If $f_{1}(x)=f_{2}(x)=\exp \left(-x^{2} / 2\right)$, then axially symmetric result is trivial, $R_{0}(\rho)=\exp \left(-\rho^{2} / 2\right)$.

We were lucky to find another example of completely symmetric self $2 \mathrm{D}$ MFT function, with eigenvalue $\Lambda=+1$ :

$$
C_{00}(\rho)=\frac{1}{2 \pi} \int_{0}^{2 \pi} c_{0}(\rho \cos \alpha) c_{0}(\rho \sin \alpha) d \alpha, \quad \Lambda=1,
$$

where $c_{0}(x)=1 / \cosh (x \sqrt{\pi / 2})$. Graph of this new function is presented on Fig 1.

Fig. 1. Self-Fourier transform function $C_{00}(\rho)$.

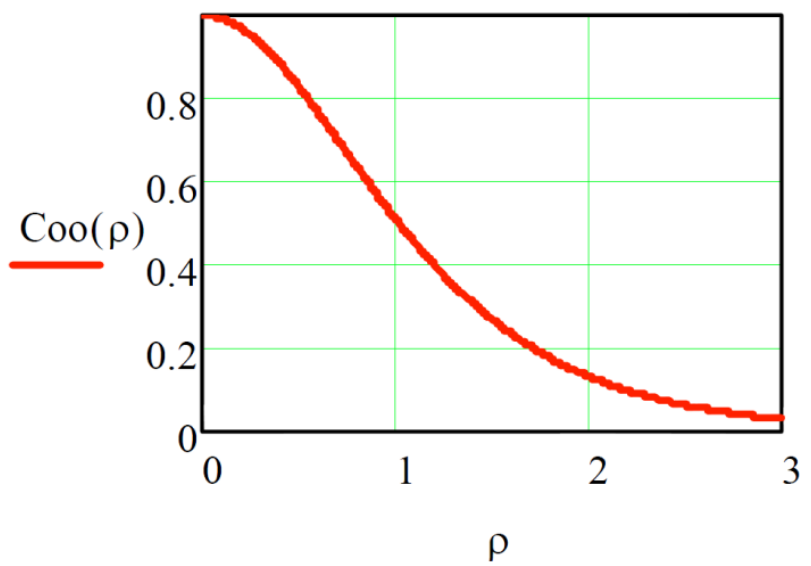

Behavior of this function at small and large $\rho$ (remember that $\rho$ is dimensionless) is as follows:

$$
C_{00}(\rho)=1-\frac{\pi}{4} \rho^{2}+O\left(\rho^{4}\right) \text { at } \rho \rightarrow 0,
$$

Normalized (by $\int_{0}^{\infty}|f(x)|^{2} \rho d \rho$ ) function $C_{00}(\rho)$ is mostly very similar to normalized axially-symmetric function $g_{0}(\rho)=$ $=\exp \left(-\rho^{2} / 2\right)$. Indeed,

$$
\frac{\left|\int_{0}^{\infty} C_{00}(\rho) g_{00}(\rho) \rho d \rho\right|^{2}}{\int_{0}^{\infty}\left[C_{00}(\rho)\right]^{2} \rho d \rho \cdot \int_{0}^{\infty}\left[g_{00}(\rho)\right]^{2} \rho d \rho}=0.992 .
$$

However, the asymptotic behavior of $C_{00}(\rho)$ at $\rho \rightarrow \infty$ is radically different from that of Gaussian function. In this respect $C_{00}(\rho)$ is a better approximation of the radial profile of a singlemode step-profile dielectric fiber with low $\mathrm{V}$-number.

In particular, consider the axially symmetric mode $L P_{01}(r)$ of a single-mode fiber with core radius $a$ and $V$-number

$$
V=(2 \pi a / \lambda)\left(n_{\text {core }}{ }^{2}-n_{\text {cladding }}{ }^{2}\right)^{1 / 2}
$$

being $V=1.7$ : well below the threshold $V<2.4$ of single-mode operation. Our new 2D SMFT function $C_{00}(\rho=r / u)$ has the best overlapping with $V=1.7$ normalized mode $L P_{01}(r)$ at $u=1.077 \cdot a$ and is equal to

$$
\begin{gathered}
F_{C}=\frac{\left|\int_{0}^{\infty} C_{00}(\rho=r / u) L P_{01}(r) r d r\right|^{2}}{\int_{0}^{\infty}\left[C_{00}(\rho=r / u)\right]^{2} r d r \cdot \int_{0}^{\infty}\left[L P_{01}(r)\right]^{2} r d r}=0.9986 \equiv \\
\equiv 1-1.14 \cdot 10^{-3}
\end{gathered}
$$


Meanwhile the same mode has optimum overlapping integral with Gaussian function $g_{00}(\rho)=\exp \left[-(\rho=r / u)^{2} / 2\right]$ at $u=1.076 \cdot a$; that square of overlapping equals

$$
\begin{gathered}
F_{g}=\frac{\left|\int_{0}^{\infty} g_{00}(\rho=r / u) L P_{01}(r) r d r\right|^{2}}{\int_{0}^{\infty}\left[g_{00}(\rho=r / u)\right]^{2} r d r \cdot \int_{0}^{\infty}\left[L P_{01}(r)\right]^{2} r d r}=0.9855 \equiv \\
\equiv 1-1.45 \cdot 10^{-2} .
\end{gathered}
$$

At $V=2.4$ (threshold value of $V$, below which single mode exists only) Gaussian function $g_{00}(\rho)$ has some advantage over $C_{00}(\rho)$ :

$$
F_{g, \text { optimum }}=1-3.3 \cdot 10^{-3}, F_{C, \text { optimum }}=1-5.0 \cdot 10^{-3} .
$$

However, both approximations are pretty good. Detailed study of approximation for fundamental modes of a fiber by Gaussian function was done by D. Marcuse in [10], where he considered a variety of smoothed profiles of fiber refractive index.

\section{Calculation of data for Beam Propagation Product (BPP) according to 21 possible criteria for 6 particular high-quality beams.}

We consider numerous possible criteria of the beam width, be it in the near field waist (in units of meters), or in the far field (in units of radians). Here is the list of 21 criteria covered, formulated for quantity of dimensions [meters]. Similar definitions are to be taken for $\theta_{x}$ or for $\theta=\left(\theta_{x}^{2}+\theta_{y}^{2}\right)^{1 / 2}$ of dimensions [radian].

1. $\Delta x$ (HWHIM): Half Width at the level Half of the Intensity at Maximum.

2. $\Delta x$ (HWe $\left.e^{-1} \mathrm{IM}\right):$ Half Width at the level $e^{-1} \equiv 0.368$ of the Intensity at Maximum.

3. $\Delta x$ (HWe $\left.e^{-2} \mathrm{IM}\right)$ : Half Width at the level $e^{-2} \equiv 0.135$ of the Intensity at Maximum

4. $\Delta x$ (HW10 $\left.{ }^{-2} \mathrm{IM}\right)$ : Half Width at the level $10^{-2}$ of the Intensity at Maximum

5. $r$ (PIB $f=0.5)$ : radius of a circle containing fraction $f=0.5$ of total Power In the Bucket of that radius.

6. $r$ (PIB $f=0.75$ ): radius of a circle containing fraction $f=0.75$ of total Power In the Bucket of that radius.

7. $r$ (PIB $f=0.865$ ): radius of a circle containing fraction $f=0.865=1-e^{-2}$ of total Power In the Bucket of that radius.

8. $r$ (PIB $f=0.9$ ): radius of a circle containing fraction $f=0.9$ of total Power In the Bucket of that radius.

9. $r$ (PIB $f=0.95)$ : radius of a circle containing fraction $f=0.95$ of total Power In the Bucket of that radius.

10. $r$ (PIB $f=0.975$ ): radius of a circle containing fraction $f=0.975$ of total Power In the Bucket of that radius.

11. $r$ (PIB $f=0.99$ ): radius of a circle containing fraction $f=0.99$ of total Power In the Bucket of that radius.

12. $s$ (PIS $f=0.5$ ): half width of the minimum width of the slit, containing fraction $f=0.5$ of total Power In that Slit of total width $2 s$.

13. $s$ (PIS $f=0.75$ ): half width of the minimum width of the slit, containing fraction $f=0.75$ of total Power In that Slit of total width $2 s$.

14. $s$ (PIS $f=0.865$ ): half width of the minimum width of the slit, containing fraction $f=0.865$ of total Power In that Slit of total width $2 s$.
15. $s$ (PIS $f=0.9$ ): half width of the minimum width of the slit, containing fraction $f=0.9$ of total Power In that Slit of total width $2 s$.

16. $s$ (PIS $f=0.95$ ): half width of the minimum width of the slit, containing fraction $f=0.95$ of total Power In that Slit of total width $2 s$.

17. $s$ (PIS $f=0.975$ ): half width of the minimum width of the slit, containing fraction $f=0.975$ of total Power In that Slit of total width $2 s$.

18. $s$ (PIS $f=0.99$ ): half width of the minimum width of the slit, containing fraction $f=0.99$ of total Power In that Slit of total width $2 s$.

19. $x_{\mathrm{rms}}=\sqrt{\left\langle(x-\bar{x})^{2}\right\rangle}$, root mean square of variation of $\mathrm{x}^{-}$ coordinate.

20. $x_{1}=\langle|x-\bar{x}|\rangle$, average modulus of variation of $\mathrm{x}^{-}$ coordinate.

21. $x_{0.5}=\left(\left\langle|x-\bar{x}|^{1 / 2}\right\rangle\right)^{2}$, square of average of square root of the modulus of coordinate variation.

We calculated the data for six different profiles of the field in the near-field zone: 1) Gaussian $E(x, y)=\exp \left[-\left(x^{2}+y^{2}\right) / w^{2}\right]$, 2) Super-Gaussian $E(x, y)=\exp \left[-\left(x^{2}+y^{2}\right)^{2} / w^{4}\right]$, 3) axiallysymmetric $2 \mathrm{D}$ sech profile: Self-Fourier Transform profile found in this work, $E(x, y)=C_{00}\left(\rho=\sqrt{x^{2}+y^{2}} / u\right)$; 4) profile of axially-symmetric mode of a single-mode fiber with $V=2.4$ and of core radius $a, E(x, y)=L P_{01}\left(r=\sqrt{x^{2}+y^{2}}\right)$; 5) Round Top Hat profile $E(x, y)=1$ at $\sqrt{x^{2}+y^{2}} \leq w, E(x, y)=0$ otherwise; 6) Factorized Hyperbolic secant profile, $E(x, y)=c_{0}(X=$ $x / u) c_{0}(Y=y / u), c_{0}(t)=1 / \cosh (t \sqrt{\pi / 2})$. They are presented in Table 1.

Since the table contains dimensionless numbers, clarification should be made, in what units of dimensions of meters those data are given. For Gaussian and Super-Gaussian beams, 1 and 2, the data are given in units of traditional notations of $w$, where $w=\Delta x\left(\mathrm{HW} e^{-2} \mathrm{IM}\right)$. For new self-Fourier-Transform function $E(x, y)=C_{00}\left(\rho=\sqrt{x^{2}+y^{2}} / u\right)$, defined by eq. (40), coordinate width is given units $u$. The parameter $u$ in $C_{00}\left(\rho=\sqrt{x^{2}+y^{2}} / u\right)$ coincides with the $\Delta x($ HW0.2622IM $)$ of the said beam, so that $\left|C_{00}(\rho=1)\right|^{2}=0.2622$, while $\mid C_{00}(\rho=$ $0)\left.\right|^{2}=1$.

For the mode of step-profile fiber with $\mathrm{V}$-number $V=2.4$ the data are given in units of core radius $a$. Finally, for factorized hyperbolic-secant $E(x, y)=c_{0}(X=x / u) c_{0}(Y=y / u), \quad c_{0}(t)=$ $1 / \cosh (t \sqrt{\pi / 2})$ parameter $u$ may be considered as $\Delta x$ (HW0.2788IM).

As for the angular profile corresponding to those beams, their parameters, like $\delta \theta$ [radians] are expressed in units $(\lambda / w)$ for 1$)$ Gaussian, 2) Super-Gaussian and 5) Round Top Hat beams; in units $(\lambda / u)$ for 3) axially-symmetric sech-beam and for 6) factorized sech-beam; for 4) $L P_{01}$-mode of a fiber with $V$-nubmer $V=2.4$ angular width is expressed in units $\lambda / a$.

Round top Hat beam \#5 has well-known angular distribution of amplitude and intensity:

$$
\left|G\left(\theta_{x}, \theta_{y}\right)\right|^{2}=\operatorname{const}\left(\frac{J_{1}(v)}{v}\right)^{2}, v=\frac{2 \pi w}{\lambda} \cdot \sqrt{\theta_{x}^{2}+\theta_{y}^{2}}
$$

so that 1-st zero of intensity of so-called "Airy disk" corresponds to $\left(\theta_{x}^{2}+\theta_{y}^{2}\right)^{0.5}=1.22 \lambda / 2 w$. Power-in-the-bucket fraction for the intensities profile (46) is given by:

$$
f(\theta)=1-\left[J_{0}\left(\frac{2 \pi w \theta}{\lambda}\right)\right]^{2}-\left[J_{1}\left(\frac{2 \pi w \theta}{\lambda}\right)\right] .
$$


Here and in Eq. (46) $J_{0}$ and $J_{1}$ are Bessel functions. Fraction of power in the bucket of the radius $\theta_{\text {Airy }}=1.22 \lambda /(2 w)$ is $f(\operatorname{PIB} \theta=1.22 \lambda /(2 w))$; numerically it is equal to $f=1-$ the $\left\langle\theta_{x}^{2}\right\rangle$ for that table is calculated by truncation of integral for $\left\langle\theta_{x}^{2}\right\rangle$ at value $\theta_{\max }=10 \lambda / w$.

Table 2 contains the values of Beam Propagation Products (BPP) for those 6 beams: $\Delta \theta \cdot \Delta x / \lambda$ or $\delta \theta \cdot r / \lambda$. In these BPP we assumed one and the same criterion (out of 21) for coordinate size ( $\Delta x$ or $r$ ) and for angular size $\left(\Delta \theta_{x}\right.$ or $\theta$ ). In principle one can compile $21 \times 21 \times 6=2646$ products, if different criteria are used for
$\left(J_{0}(3.8317)\right)^{2}=0.8378$. Intensity wings of this angular distribution yield logarithmically divergent $\left\langle\theta_{x}^{2}\right\rangle$. Finite value of

near field and for far field; Table 1 contains all the necessary data. Table 3 follows the ideology form Lantigua et al. [7]: to divide BPP of measured beam by BPP of Gaussian beam, taken by the same criteria. In Lantigua et. al. authors used experimentally measured coordinate and angular width taken by particular criterion PIS $f=0.85$ (which is very close to our $\left.0.865=1-e^{-2}\right)$.

Table 1: Calculation of individual widths of various beams according to various criteria, see text.

\begin{tabular}{|c|c|c|c|c|c|c|}
\hline & 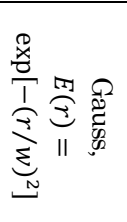 & 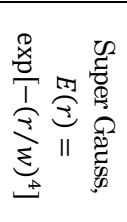 & 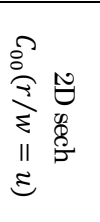 & 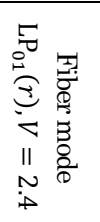 & 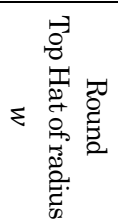 & 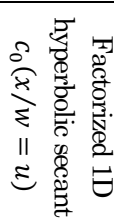 \\
\hline$\Delta x$ HWHIM, [width/w] & 0.5887 & 0.7677 & 0.6930 & 0.6840 & 1 & 0.7032 \\
\hline$\Delta x \mathrm{HW} e^{-1} \mathrm{IM},[$ width/w] & 0.7070 & 0.8409 & 0.8476 & 0.8052 & 1 & 0.8657 \\
\hline$\Delta x \mathrm{HW} e^{-2} \mathrm{IM},[$ width/w] & 1 & 1 & 1.2685 & 1.0699 & 1 & 1.3225 \\
\hline$\Delta x$ HW10 ${ }^{-2} \mathrm{IM},[$ width $/ w]$ & 1.5170 & 1.2318 & 2.1956 & 1.6923 & 1 & 2.3882 \\
\hline$\theta_{x}$ HWHIM, angle $\cdot(w / \lambda)$ & 0.1874 & 0.2447 & 0.1099 & 0.1493 & 0.2572 & 0.1119 \\
\hline$\theta_{x} \mathrm{HW} e^{-1} \mathrm{IM}$, angle $\cdot(w / \lambda)$ & 0.2250 & 0.2913 & 0.1345 & 0.1825 & 0.3048 & 0.1378 \\
\hline$\theta_{x} \mathrm{HW} e^{-2} \mathrm{IM}$, angle $\cdot(w / \lambda)$ & 0.3183 & 0.3993 & 0.2012 & 0.2724 & 0.4112 & 0.2105 \\
\hline$\theta_{x} \mathrm{HW} 10^{-2} \mathrm{IM}$, angle $\cdot(w / \lambda)$ & 0.4829 & 0.5499 & 0.3483 & 0.4518 & 0.5442 & 0.3801 \\
\hline$r \mathrm{PIB} \mathrm{f}=0.5,[$ width $/ w]$ & 0.5887 & 0.5807 & 0.7830 & 0.6394 & 0.7070 & 0.7848 \\
\hline$r \mathrm{PIB} \mathrm{f}=0.75,[$ width $/ w]$ & 0.8326 & 0.7584 & 1.1551 & 0.8893 & 0.8660 & 1.1591 \\
\hline$r \mathrm{PIB} \mathrm{f}=0.865,[$ width $/ w]$ & 1.0006 & 0.8641 & 1.4370 & 1.0699 & 0.9299 & 1.4450 \\
\hline$r$ PIB $\mathrm{f}=0.9,[$ width $/ w]$ & 1.0730 & 0.9069 & 1.5677 & 1.1574 & 0.9487 & 1.5765 \\
\hline$r$ PIB $\mathrm{f}=0.95,[$ width $/ w]$ & 1.2239 & 0.99 & 1.8547 & 1.3573 & 0.9747 & 1.8686 \\
\hline$r$ PIB $\mathrm{f}=0.975,[$ width $/ w]$ & 1.3581 & 1.0586 & 2.1304 & 1.5559 & 0.9874 & 2.1509 \\
\hline$r$ PIB $\mathrm{f}=0.99,[$ width $/ w]$ & 1.5174 & 1.1349 & 2.4842 & 1.8161 & 0.9950 & 2.5158 \\
\hline$\theta \mathrm{PIB} \mathrm{f}=0.5$, angle $\cdot(w / \lambda)$ & 0.1874 & 0.2327 & 0.1246 & 0.1654 & 0.2654 & 0.1249 \\
\hline$\theta \mathrm{PIB} \mathrm{f}=0.75$, angle $\cdot(w / \lambda)$ & 0.2650 & 0.3237 & 0.1838 & 0.2414 & 0.3917 & 0.1845 \\
\hline$\theta$ PIB $\mathrm{f}=0.865$, angle $\cdot(w / \lambda)$ & 0.3185 & 0.3838 & 0.2287 & 0.2963 & 0.7766 & 0.2300 \\
\hline$\theta \mathrm{PIB} \mathrm{f}=0.9$, angle $\cdot(w / \lambda)$ & 0.3415 & 0.4095 & 0.2495 & 0.3208 & 0.9063 & 0.2509 \\
\hline$\theta \mathrm{PIB} \mathrm{f}=0.95$, angle $\cdot(w / \lambda)$ & 0.3896 & 0.4638 & 0.2952 & 0.3720 & 1.7802 & 0.2974 \\
\hline$\theta \mathrm{PIB} \mathrm{f}=0.975$, angle $\cdot(w / \lambda)$ & 0.4323 & 0.5203 & 0.3391 & 0.4175 & 2.9031 & 0.3423 \\
\hline$\theta \mathrm{PIB} \mathrm{f}=0.99$, angle $\cdot(w / \lambda)$ & 0.4830 & 0.8080 & 0.3954 & 0.4705 & 5.1887 & 0.4004 \\
\hline$s$ PIS f $=0.5,[$ width $/ w]$ & 0.3372 & 0.3357 & 0.4488 & 0.3684 & 0.4040 & 0.4383 \\
\hline$s$ PIS $\mathrm{f}=0.75,[$ width $/ w]$ & 0.5752 & 0.5423 & 0.7864 & 0.6220 & 0.6347 & 0.7763 \\
\hline$s$ PIS $\mathrm{f}=0.865$, [width/ $w$ ] & 0.7467 & 0.6727 & 1.0498 & 0.8031 & 0.7607 & 1.0465 \\
\hline$s$ PIS $\mathrm{f}=0.9,[$ width $/ w]$ & 0.8224 & 0.7257 & 1.1724 & 0.8845 & 0.8054 & 1.1747 \\
\hline$s$ PIS $\mathrm{f}=0.95,[$ width $/ w]$ & 0.9800 & 0.8283 & 1.4425 & 1.0652 & 0.8783 & 1.4615 \\
\hline$s$ PIS $\mathrm{f}=0.975$, [width $/ w]$ & 1.1207 & 0.9121 & 1.7024 & 1.2463 & 0.9237 & 1.7431 \\
\hline$s$ PIS f $=0.99,[$ width $/ w]$ & 1.2880 & 1.0037 & 2.0371 & 1.4860 & 0.9587 & 2.1117 \\
\hline$\theta_{s} \mathrm{PIS} \mathrm{f}=0.5$, angle $\cdot(w / \lambda)$ & 0.1073 & 0.1335 & 0.0714 & 0.0946 & 0.1590 & 0.0697 \\
\hline$\theta_{s}$ PIS $\mathrm{f}=0.75$, angle $\cdot(w / \lambda)$ & 0.1831 & 0.2257 & 0.1252 & 0.1647 & 0.2838 & 0.1235 \\
\hline$\theta_{s} \mathrm{PIS} \mathrm{f}=0.865$, angle $\cdot(w / \lambda)$ & 0.2377 & 0.2907 & 0.1671 & 0.2179 & 0.4194 & 0.1665 \\
\hline$\theta_{s}$ PIS $\mathrm{f}=0.9$, angle $\cdot(w / \lambda)$ & 0.2618 & 0.3190 & 0.1866 & 0.2420 & 0.6093 & 0.1869 \\
\hline$\theta_{s}$ PIS $\mathrm{f}=0.95$, angle $\cdot(w / \lambda)$ & 0.3119 & 0.3782 & 0.2296 & 0.2935 & 1.1172 & 0.2326 \\
\hline$\theta_{s} \mathrm{PIS} \mathrm{f}=0.975$, angle $\cdot(w / \lambda)$ & 0.3567 & 0.4343 & 0.2709 & 0.3403 & 1.9249 & 0.2774 \\
\hline$\theta_{s}$ PIS $\mathrm{f}=0.99$, angle $\cdot(w / \lambda)$ & 0.4100 & 0.5308 & 0.3242 & 0.3966 & 3.7706 & 0.3361 \\
\hline$\left\langle x^{2}\right\rangle^{\frac{1}{2}}$, meter $/ w$ & 0.5 & 0.4465 & 0.7193 & 0.5509 & 0.5 & 0.7236 \\
\hline$\langle|x|\rangle$, meter $/ w$ & 0.3989 & 0.3701 & 0.5564 & 0.4363 & 0.4244 & 0.5530 \\
\hline$(\langle\sqrt{|x|}\rangle)^{2}$, meter $/ w$ & 0.3380 & 0.3205 & 0.4641 & 0.3693 & 0.3723 & 0.4586 \\
\hline$\left\langle\theta_{x}{ }^{2}\right\rangle^{\frac{1}{2}}$, angle $\cdot(w / \lambda)$ & 0.1591 & 0.2011 & 0.1145 & 0.1474 & 0.7182 & 0.1152 \\
\hline$\left\langle\left|\theta_{x}\right|\right\rangle$, angle $\cdot(w / \lambda)$ & 0.1270 & 0.1581 & 0.0885 & 0.1154 & 0.3148 & 0.0880 \\
\hline
\end{tabular}


Table 2. Beam propagation products for various near-field profiles $E(x, y)$; see text.

\begin{tabular}{|c|c|c|c|c|c|c|}
\hline $\begin{array}{c}r \cdot \theta / \lambda \\
s \cdot \theta_{x} / \lambda \\
M\end{array}$ & 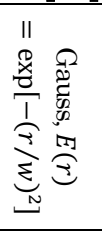 & 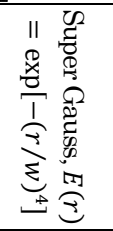 & 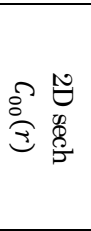 & 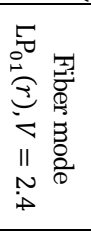 & 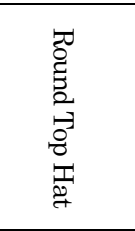 & 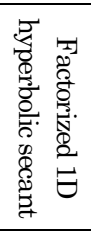 \\
\hline HWHIM & 0.1103 & 0.1878 & 0.0764 & 0.1021 & 0.2572 & 0.0787 \\
\hline $\mathrm{HW} e^{-1} \mathrm{IM}$ & 0.1591 & 0.2450 & 0.1143 & 0.1469 & 0.3048 & 0.1193 \\
\hline $\mathrm{HW} e^{-2} \mathrm{IM}$ & 0.3183 & 0.3993 & 0.2561 & 0.2915 & 0.4112 & 0.2783 \\
\hline $\mathrm{HW} 10^{-2} \mathrm{IM}$ & 0.7329 & 0.6774 & 0.7672 & 0.7645 & 0.5443 & 0.9078 \\
\hline $\operatorname{PIB} f=0.5$ & 0.1103 & 0.1351 & 0.0976 & 0.1058 & 0.1877 & 0.0980 \\
\hline $\operatorname{PIB} f=0.75$ & 0.2206 & 0.2455 & 0.2123 & 0.2147 & 0.3392 & 0.2138 \\
\hline $\operatorname{PIB} f=0.865$ & 0.3183 & 0.3316 & 0.3286 & 0.3170 & 0.7221 & 0.3323 \\
\hline $\operatorname{PIB} f=0.9$ & 0.3665 & 0.3714 & 0.3911 & 0.3713 & 0.8598 & 0.3955 \\
\hline $\operatorname{PIB} f=0.95$ & 0.4768 & 0.4591 & 0.5475 & 0.505 & 1.7352 & 0.5557 \\
\hline $\mathrm{PIB} f=0.975$ & 0.5871 & 0.5508 & 0.7223 & 0.6496 & 2.8666 & 0.7363 \\
\hline $\operatorname{PIB} f=0.99$ & 0.7329 & 0.9169 & 0.9822 & 0.8544 & 5.1627 & 1.0073 \\
\hline PIS $f=0.5$ & 0.0362 & 0.0448 & 0.0320 & 0.0348 & 0.0642 & 0.0306 \\
\hline $\operatorname{PIS} f=0.75$ & 0.1053 & 0.1224 & 0.0985 & 0.1025 & 0.1801 & 0.0959 \\
\hline $\operatorname{PIS} f=0.865$ & 0.1775 & 0.1955 & 0.1754 & 0.1750 & 0.3191 & 0.1743 \\
\hline $\operatorname{PIS} f=0.9$ & 0.2153 & 0.2315 & 0.2188 & 0.2141 & 0.4907 & 0.2196 \\
\hline $\operatorname{PIS} f=0.95$ & 0.3057 & 0.3133 & 0.3312 & 0.3126 & 0.9812 & 0.3400 \\
\hline $\operatorname{PIS} f=0.975$ & 0.3998 & 0.3962 & 0.4613 & 0.4241 & 1.7781 & 0.4836 \\
\hline $\operatorname{PIS} f=0.99$ & 0.5280 & 0.5327 & 0.6605 & 0.5893 & 3.6150 & 0.7097 \\
\hline$M_{x}^{2}=(4 \pi / \lambda) \cdot \sqrt{\left\langle x^{2}\right\rangle\left\langle\theta_{x}^{2}\right\rangle}$ & 1 & $\begin{array}{l}2 / \sqrt{\pi}= \\
=1.1281\end{array}$ & 1.0349 & 1.0182 & $4.5771(\infty)$ & 1.0472 \\
\hline$M_{1}=(4 \pi / \lambda) \cdot\langle|x|\rangle\left\langle\left|\theta_{x}\right|\right\rangle$ & 0.6366 & 0.7349 & 0.6192 & 0.6326 & 1.6784 & 0.6117 \\
\hline$M_{0.5}=(4 \pi / \lambda)\langle\sqrt{|x|}\rangle^{2}\left\langle\sqrt{\left|\theta_{x}\right|}\right\rangle^{2}$ & 0.4569 & 0.5381 & 0.4308 & 0.4493 & 0.9787 & 0.4206 \\
\hline
\end{tabular}

Table 3. The ratios of beam propagation products for the beams under study to those of Gaussian beam. We have highlighted the particular cells of that table where those ratios are smaller than 1 . We see that completely symmetric self-Fourier-Transformed beam $C_{00}(\rho)$ based on hyperbolic secant functions (Eq. (40)) yields certain advantage over the Gaussian beam, albeit for a limited number of criteria. Actually, that advantage is rather modest, about $4 \%$ to $30 \%$, depending on particular criterion.

\begin{tabular}{|c|c|c|c|c|c|c|}
\hline $\begin{array}{c}r \cdot \theta / \lambda \\
s \cdot \theta_{x} / \lambda \\
M\end{array}$ & 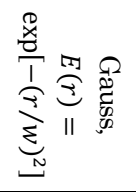 & 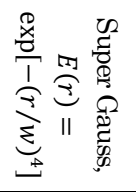 & 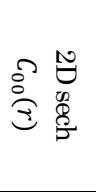 & 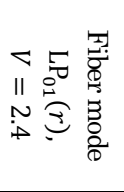 & 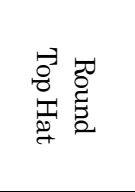 & 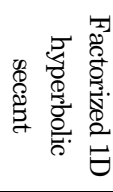 \\
\hline HWHIM & 1 & 1.7026 & 0.6927 & 0.9257 & 2.3318 & 0.7135 \\
\hline $\mathrm{HW} e^{-1} \mathrm{IM}$ & 1 & 1.5399 & 0.7184 & 0.9233 & 1.9158 & 0.7497 \\
\hline $\mathrm{HW} e^{-2} \mathrm{IM}$ & 1 & 1.2545 & 0.8046 & 0.9158 & 1.2919 & 0.8744 \\
\hline $\mathrm{HW} 10^{-2} \mathrm{IM}$ & 1 & 0.9243 & 1.0468 & 1.0431 & 0.7427 & 1.2392 \\
\hline $\mathrm{PIB} f=0.5$ & 1 & 1.2248 & 0.8849 & 0.9592 & 1.7017 & 0.8887 \\
\hline $\operatorname{PIB} f=0.75$ & 1 & 1.1129 & 0.9624 & 0.9733 & 1.5376 & 0.9693 \\
\hline $\operatorname{PIB} f=0.865$ & 1 & 1.0418 & 1.0324 & 0.9959 & 2.2686 & 1.0440 \\
\hline $\mathrm{PIB} f=0.9$ & 1 & 1.0134 & 1.0671 & 1.0131 & 2.346 & 1.0793 \\
\hline $\operatorname{PIB} f=0.95$ & 1 & 0.9629 & 1.1483 & 1.0591 & 3.6393 & 1.1655 \\
\hline $\operatorname{PIB} f=0.975$ & 1 & 0.9382 & 1.2303 & 1.1065 & 4.8826 & 1.2541 \\
\hline $\operatorname{PIB} f=0.99$ & 1 & 1.2511 & 1.3402 & 1.1658 & 7.0442 & 1.3744 \\
\hline PIS $f=0.5$ & 1 & 1.2376 & 0.884 & 0.9613 & 1.7735 & 0.8445 \\
\hline $\operatorname{PIS} f=0.75$ & 1 & 1.1624 & 0.9354 & 0.9734 & 1.7104 & 0.9108 \\
\hline PIS $f=0.865$ & 1 & 1.1014 & 0.9882 & 0.9859 & 1.7977 & 0.9820 \\
\hline PIS $f=0.9$ & 1 & 1.0752 & 1.0163 & 0.9944 & 2.2791 & 1.0200 \\
\hline $\operatorname{PIS} f=0.95$ & 1 & 1.0249 & 1.0834 & 1.0226 & 3.2097 & 1.1121 \\
\hline $\operatorname{PIS} f=0.975$ & 1 & 0.991 & 1.1538 & 1.0608 & 4.4475 & 1.2095 \\
\hline PIS $f=0.99$ & 1 & 1.0089 & 1.2509 & 1.1161 & 6.8466 & 1.3440 \\
\hline$(4 \pi / \lambda) \cdot \sqrt{\left\langle x^{2}\right\rangle\left\langle\theta_{x}^{2}\right\rangle}$ & 1 & 1.1281 & 1.0349 & 1.0182 & $4.5771(\infty)$ & 1.0472 \\
\hline
\end{tabular}


$M_{1}=(4 \pi / \lambda) \cdot\langle|x|\rangle\left\langle\left|\theta_{x}\right|\right\rangle$
$M_{0.5}=(4 \pi / \lambda)\langle\sqrt{|x|}\rangle^{2}\left\langle\sqrt{\left|\theta_{x}\right|}\right\rangle$

The results depicted in Table 3 disprove a deeply entrenched myth that Gaussian field profile has the best BPP. This myth is definitely valid for r.m.s. criterion (i.e. $M_{x}^{2}$ criterion), but not necessarily for other criteria. Particular boxes where other beams show BPP smaller than Gaussian are highlighted. However that "advantage" of other beams is not very strong.

Observing the data from Tables 1,2 and 3, we see that 6 beams of essentially diffraction quality all have BPP about 1 . Therefore particular choice of criteria should depend on the task for which the beam is intended in a particular application. Experimental work by Lantigua et. al. used (PIS $f=0.85$ ) criteria both for near field and for far field zones. Power-In-theSlit is easier to measure in experiment than PIB, Power In the circular Bucket. On the other hand, PIB may be more important in a number of applications of laser beams.

\section{Conclusion}

We discussed 21 different criteria of width of the laser beam. Those criteria are applicable both for near-field waist, where the width $\Delta x$ or $r$ has dimensions [meters], and for far field zone, where width $\Delta \theta_{x}$ or $\theta$ has dimensions of [radians]. Since field amplitude in the far-field zone is a Fourier Transform (FT) (2) or (3) of the profile of the field in the waist, we provide the necessary information about properties of FT in Physical approach (PFT), Eq. (4), in Mathematical one (MFT), Eq. (7) and computationally convenient Discrete Fourier Transform (DFT), Eq. (25). We established simple quantitative relationships between PFT, MFT and DFT.

That information has allowed us to find axially-symmetric eigenfunction of MFT Eq. (40).

Using Fourier Transformation, we were able to find the values of $\Delta x$ and $\Delta \theta_{x}$ (or $r$ and $\theta$ ) according to 21 criteria for slightly different beams of almost diffraction quality.

In our opinion, the use of particular criterion "width of the slit, containing $85 \%$ of total power", constitutes a reasonable compromise between following the energy budget of the beam, on one hand, and suppression of unimportant wings of intensity distribution and measurement noise, on the other hand. Such technique has been demonstrated in recent physical experiments [7]. Dividing beam propagation product to that of ideal Gaussian beam provides, quality parameter, which is close to well-known $M_{x}^{2}$-criterion, but without the drawbacks of the latter.

Results of our theoretical work show that taking some other diffraction-quality beam as etalon for comparison (instead of Gaussian) does not introduce much of a change.

In Appendices A and B we further illustrate important properties of PFT, and connection between DFT and MFT.

Acknowledgments. The work was supported by Navy Contract N68335-12-C-0239 and HEL JTO, ARO contract W911NF-10-10441.

\section{Appendix A. Notion of edge waves: asymptotic behavior of Fourier-transform at large " $q$ ".}

Fraunhofer zone, i.e. far-field amplitude may be presented in the following form (see Goodman [11,12] and Gbur [13])

$$
\begin{gathered}
E(X, Y, Z)=\frac{k}{2 \pi i Z} \exp (i k|\boldsymbol{R}|) G_{2}\left(q_{x}, q_{y}\right), \\
G_{2}(\boldsymbol{q})=\iint d x^{\prime} d y^{\prime} E\left(z^{\prime}=0, \boldsymbol{r}^{\prime}\right) \exp \left(-i \boldsymbol{q} \cdot \boldsymbol{r}^{\prime}\right) .
\end{gathered}
$$

Here $\left(q_{x}, q_{y}\right)=\left(\theta_{x}, \theta_{y}\right)(2 \pi / \lambda)=(X / Z, Y / Z)(2 \pi / \lambda)$, and we assume $\exp (-i \omega t)$ time dependence.

It means that the angular-dependent diffraction amplitude is $2 \mathrm{D}$-Fourier transform of the original field. This Appendix A is devoted to discussion of the properties of $1 \mathrm{D}$-Fourier transform,

$$
G(q)=G_{2}\left(q_{x}=q, q_{y}=0\right)=\int_{-\infty}^{+\infty} E(x) \exp (-i q x) d x
$$

Here

$$
E(x)=\int_{-\infty}^{+\infty} E\left(x, y^{\prime}\right) d y^{\prime}
$$

We assume that $E(x \rightarrow-\infty)=E(x \rightarrow+\infty)=0$. Consider the question of asymptotic behavior of diffraction amplitude, i.e. 1DFourier transformation (A2) at large values of $|q|$ [actual small parameter of this asymptotic expansion is $(|q| \Delta x)^{-1}$. With this aim in mind, one can identically transform $A(q)$ from (A2) to

$$
G(q) \equiv \frac{i}{q} \int_{-\infty}^{+\infty} E(x) \frac{d}{d x} \exp (-i q x) d x
$$

Integration of this formula by parts, with account of $E(x \rightarrow-\infty)=E(x \rightarrow+\infty)=0$, yields:

$$
G(q) \equiv-\frac{i}{q} \int_{-\infty}^{+\infty} \exp (-i q x) \frac{d}{d x} E(x) d x .
$$

If function $E(x)$ contains several discrete steps at points $x=a, \quad x=b, \quad$ with the magnitude of steps $\Delta E_{A}=$ $=E(x \rightarrow a+\varepsilon)-E(x \rightarrow a-\varepsilon), \varepsilon \rightarrow+0$, etc., then the function $d E / d x$ contains corresponding number of $\delta$-functions

$\frac{d E}{d x}=\Delta E_{a} \cdot \delta(x-a)+\Delta E_{b} \cdot \delta(x-b)+\cdots+\left(\frac{d E}{d x}\right)_{\text {extracted }}$.

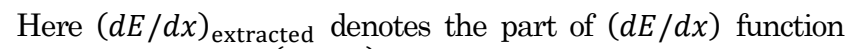
with extracted $\Delta E_{j} \cdot \delta\left(x-x_{j}\right)$ terms. As a result, $G(q)$ takes the form

$$
\begin{aligned}
G(q)= & -\frac{i}{q}\left[\Delta E_{a} e^{-i q a}+\Delta E_{b} e^{-i q b}+\cdots\right]- \\
& -\frac{i}{q} \int_{-\infty}^{+\infty} e^{-i q x}\left(\frac{d E}{d x}\right)_{\text {extracted }} d x .
\end{aligned}
$$

Under application of the same procedure several times to $(d E / d x)_{\text {extracted }}$, one gets the asymptotic expansion of Fourier transform amplitude $G(q)$ in the form

$$
\begin{gathered}
G(q)=\left(-\frac{i}{q}\right)\left[\Delta E_{a} e^{-i q a}+\Delta E_{b} e^{-i q b}+\cdots\right]+ \\
+\left(-\frac{i}{q}\right)^{2}\left[\Delta\left(\frac{d E}{d x}\right)_{a} e^{-i q a}+\Delta\left(\frac{d E}{d x}\right)_{b} e^{-i q b}+\cdots\right]+
\end{gathered}
$$




$$
+\left(-\frac{i}{q}\right)^{3}\left[\Delta\left(\frac{d^{2} E}{d x^{2}}\right)_{a} e^{-i q a}+\Delta\left(\frac{d^{2} E}{d x^{2}}\right)_{b} e^{-i q b}+\cdots\right]+\cdots
$$

Surprisingly, we have not seen analog of expansion (A8) in any mathematical textbook, albeit qualified people definitely do know this result. Citing Professor M. V. Berry and late Professor V. I. Arnold, "This result is well known to those, who know well"; though they used this phrase on another occasion.

There are several separate corollaries of the result (A8). Consider the function $E(x)$ which has zero limits at $x \rightarrow \pm \infty$ itself, and all its derivates have the same property. Then

1) If $E(x)$ has finite number of steps (discontinuities), then

$$
G(q)=\left(-\frac{i}{q}\right)\left[\Delta E_{a} e^{-i q a}+\Delta E_{b} e^{-i q b}+\cdots\right]+o\left(\frac{1}{q}\right) .
$$

i.e. $G(q)$ decreases as $(1 / q)$ at $|q| \rightarrow \infty$, with particular coefficient given by (A9). Graphs Fig. 2 illustrate qualitatively the structure of functions $E(x), \sum \Delta_{j} \delta\left(x-x_{j}\right)+(d E / d x)_{\text {extracted }}$, as if $E(x)$ were real function. In actual applications $E(x)$ may be complexvalued.

2) If $E(x)$ is continuous by itself, but has several discrete steps of derivative, then

$$
G(q) \doteq\left(-\frac{i}{q}\right)^{2}\left[\Delta\left(\frac{d E}{d x}\right)_{a} e^{-i q a}+\Delta\left(\frac{d E}{d x}\right)_{b} e^{-i q b}+\cdots\right] .
$$

3) If the function $E(x)$ and its derivatives: 1 st, 2 nd, ..., up to the $(\mathrm{N}-1)$ st including, are continuous, then

$$
G(q) \doteq\left(-\frac{i}{q}\right)^{N+1}\left[\Delta\left(\frac{d^{N} E(x)}{d x^{N}}\right)_{a} e^{-i q a}+\cdots\right]
$$

4) If the function $E(x)$ and all its derivatives are continuous, then $G(q)$ at $|q| \rightarrow \infty$ goes down faster than any power of $|q|$.
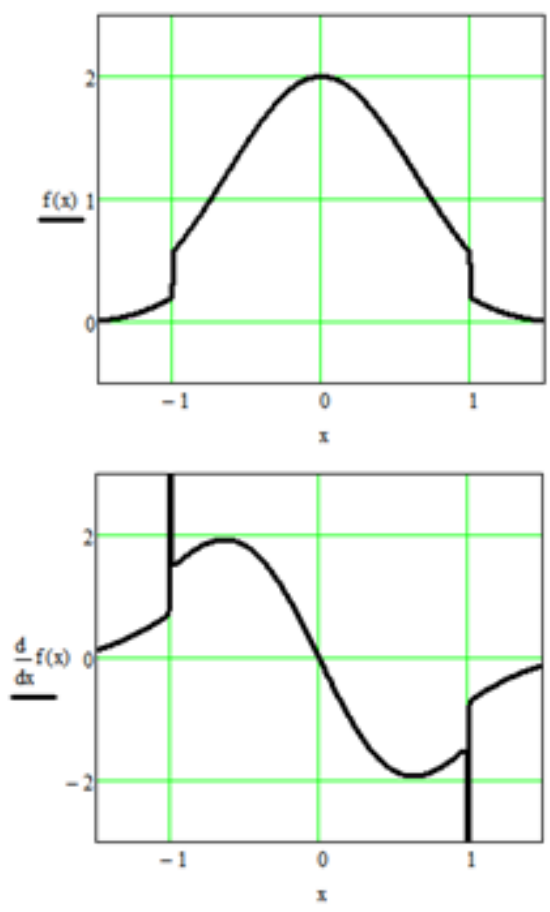

Fig. 2. Example of function possessing steps and derivative of that function with $\delta$-type singularities.
Physical reason for amplitude $E(x)$ to have discontinuity at integration plane is the presence of sharp dark edges of aperture, these edges limit the passage of the beam. The corresponding terms in $G(q)$ are "edge waves", emitted in the process of diffraction of incident wave upon that edge; compare to exact theory of Fresnel diffraction by semi-infinite plane, [14]. Contribution of steps of field derivative may be considered as resulting from diffraction by the edge of the transparent refractive prism or as a contribution from a sharp corner in an aperture.

Consider an interesting example of the function $E(x)=$ $\exp (-|x| / a)$. It is continuous by itself, but has step of derivative, and as a result

$\frac{d E}{d x}=-\frac{1}{a} \frac{x}{|x|} E(x) ; \quad \frac{d^{2} E}{d x^{2}}=-\frac{2}{a} \delta(x-0)+\left(\frac{1}{a}\right)^{2} E(x) ; \ldots$.

In this way one can write

$$
\begin{aligned}
& \frac{d^{2 k} E}{d x^{2 k}}=-\frac{2}{a} \delta(x-0) \frac{1}{a^{2 k-2}}+\left(\frac{1}{a}\right)^{2 k} E(x), \\
& G(q)=-2 a \sum_{k=0}^{\infty}\left(-\frac{1}{(q a)^{2}}\right)^{k}=\frac{2 a}{1+(q a)^{2}} .
\end{aligned}
$$

So, the summation of asymptotic series (A8) yields exact Lorentzian profile of $G(q)$.

The general statement

-"Fourier transform lives on the singularities of original" is valid even for infinitely smooth Lorentzian original,

$$
E(x)=\frac{1}{1+(x / b)^{2}},
$$

if one is allowed to consider singularities $x=i b$, and $x=-i b$ in complex plane $x=\operatorname{Re}(x)+i \operatorname{Im}(x)$. Indeed, for this function $E(x)$ contour integration in complex plane is elementary, and yields

$$
G(q)= \begin{cases}(\pi b) \exp [-i q(+i b)] & \text { for } \quad q>0 \\ (\pi b) \exp [-i q(-i b)] & \text { for } \quad q<0\end{cases}
$$

i.e.

$$
G(q)=(\pi b) \exp (-b|q|)
$$

which 1) is exact result and 2) is in agreement with the general ideology of eq. (A8):

"Fourier transform lives on the singularities of original".

Similar observation can be made for $E(x)=1 / \cosh (x / c)$, with singularities at $x= \pm i \cdot c \cdot \pi \cdot(n+1 / 2), n=0,1,2, \ldots$. Indeed, in that case

$$
E(x)=\frac{1}{\cosh (x / c)} \rightarrow G(q)=\frac{c \pi}{\cosh (q c \pi / 2)}
$$

and asymptotic behavior of $A(q)$ at large $|q|$ is

$$
G(q) \doteq c \pi \exp (-|q| c \pi / 2),
$$

due to contributions of those poles $x_{n}= \pm i \pi c(n+1 / 2)$ of original, which are closest to real axis $(n=0))$, in complete accord with ideology of eq. (A8). Moreover, the traditional way of exact calculating Fourier transform for $E(x)$ from (A16) is to elucidate the contribution of those two poles.

We also were able to find a function $f_{c}(x)$ with the following curious asymptotic behavior of MFT at $|x| \rightarrow \infty$. Its MFT decreases faster than any power of $|x|^{-n}$, but slower than $\exp (-\gamma|x|)$ with any $\gamma>0$. To possess such unusual property, 
$f_{\mathrm{c}}(x)$ must have singularity (or several of them) on real axis in complex plane, $\operatorname{Im}\left(x=x^{\prime}+i x^{\prime \prime}\right)=0$. However, this singularity should not have discontinuity of $f_{\mathrm{c}}(x)$ or of any derivative $d^{N} f / d x^{N}$ of finite order $N$. Here is an example of such function:

$$
f_{c}(x)=\left[1-\exp \left(-\frac{1}{|x|}\right)\right] \exp \left(-\sqrt[4]{\left(1+x^{2}\right)} \sqrt{2}\right) .
$$

By itself $f_{c}(x)$ is not an eigenfunction of MFT. However we were able to check numerically that application of procedure Eq. (18) from the main text to $f_{c}(x)$ transforms it to an MFT eigenfunction, while preserving property

$$
f_{S F T}(x)=\exp \left\{-c \cdot|x|^{\frac{1}{2}}+O[\ln |x|]\right\} \text { at } x \rightarrow \infty, c \approx 1.6 \text {. }
$$

However, the resultant self-MFT function at $|x| \gtrsim 1$ had oscillations (changes of sign).

\section{Appendix B}

\section{Study of eigenfunctions of MFT via DFT.}

The discussion of eigenfunctions of MFT operator acting upon functions of dimensionless argument $x$ requires large symmetric interval $-L / 2<x<L / 2$, i.e. $a=-L / 2$, and $L$ is also dimensionless. Using DFT as approximation of MFT means that step in $x$-coordinate is same as step in $q$-coordinate. Recalling that $s_{x}=L / N$ and $s_{q}=2 \pi / L$, we got from the requirement $s_{q}=s_{x}$ the relationship $2 \pi / L=L / N$, that is

$$
L=\sqrt{2 \pi N}, \quad s_{x}=s_{q}=\sqrt{2 \pi / N} .
$$

So the length $L$ of the $x$-interval $-L / 2<x<L / 2$ is dimensionless number in that application of DFT, and it grows as $\sqrt{2 \pi N}$ with the growth of $N$. For example $N=2^{10}=1024$ yields interval of dimensionless length $L \approx 80$, and step $s_{x}=$ $s_{q} \approx 0.08$; and for $N=2^{20}=1,084,574$ one gets $L \approx 2.6 \cdot 10^{3}$, $s_{x}=s_{q} \approx 2.5 \cdot 10^{-3}$. Larger $N$ yields better approximation of MFT by DFT. In its turn, approximation of

$$
h(x)=\operatorname{MFT}\{f\}(x)=\frac{1}{\sqrt{2 \pi}} \int f\left(x^{\prime}\right) \exp \left(i x x^{\prime}\right) d x
$$

via $\mathrm{DFT}$ is made by the following formulae:

$$
\begin{gathered}
f_{n}=f(-0.5 L+n \cdot L / N), \quad y_{m}=\frac{L}{N} \cdot \operatorname{phys}(m), \\
h\left(y_{m}\right)=(-1)^{m}(D F T\{f\})_{m} .
\end{gathered}
$$

Difference between $\Lambda \cdot f_{n}$ and $(-1)^{n} \cdot(\operatorname{DFT}\{f\})_{\text {phys }(n)+N / 2}$ characterizes error in hypothetical relationship

$$
f(x)=?=\Lambda \frac{1}{\sqrt{2 \pi}} \int_{-\infty}^{+\infty} f\left(x^{\prime}\right) \exp \left(i x x^{\prime}\right) d x
$$

We verified this procedure with functions $g_{0}(x)=$ $=\exp \left(-x^{2} / 2\right), c_{0}(x)=1 / \cosh (x \sqrt{\pi / 2})$, both corresponding to eigenvalue $\Lambda_{0}=1$, function $g_{1}(x)=0.5(x-d / d x) g_{0}(x) \equiv$ $\equiv x g_{0}(x)$, corresponding to eigenvalue $\Lambda_{1}=+i$. Even for very small $N, N=32, L \approx 14, s \approx 0.4$, maximum modulus of error under that procedure was $10^{-11}$ for $g_{0}(x), 10^{-10}$ for $x g_{0}(x)$ and $10^{-4}$ for $c_{0}(x)$. For $N=512, L \approx 56, s \approx 0.11$ maximum modulus of error was less or about $10^{-15}$ for all of these three functions.

\section{References:}

[1] A. E. Siegman, Lasers (University Science Books, 1986).

[2] A. E. Siegman, "How to (maybe) measure laser beam quality," in DPSS (Diode Pumped Solid State) Lasers: Applications and Issues, M. Dowley, ed., 17, OSA Trends in Optics and Photonics (OSA, 1998), paper MQ1.

[3] ISO Standard 11146, "Lasers and laser-related equipment - Test methods for laser beam widths, divergence angles and beam propagation ratios" (2005)

[4] J. M. Slater, B. Edwards. "Characterization of high-power lasers." Laser Technology for Defense and Security VI. Ed. Mark Dubinskii \& Stephen G. Post. Orlando, Florida, USA: SPIE, 2010. 76860W-12.

[5] S. Ruschin, E. Yaakobi, E. Shekel, Gaussian content as a laser beam quality parameter, Appl. Opt., August 1 2011, ; 50(22), pp. 4376-81.

[6] H. C. Miller, A laser beam quality definition based on induced temperature rise, Optics Express, Vol. 20, Issue 27, pp. 28819-28828 (2012)

[7] C. Lantigua, J. Lumeau, V. Smirnov, L. Glebov, New metric for the measurement of the quality of complex beams. (To be submitted).

[8] A. Lohmann, D. Mendlovic, Self-Fourier objects and other self-transform objects J. Opt.. Socs. Am. A, 9, pp. 2009-2012.

[9] M. J. Caola, Self-Fourier functions, J. Phys. A: Math. Gen. 24 (1991) L1143-L1144.

[10] D. Marcuse, Gaussian approximation of the fundamental modes of graded-index fiber, J. Opt. Soc. Am., Vol. 68, No. 1, January 1978, pp. 103-109.

[11] J. Goodman, Introduction to Fourier Optics (Roberts and Company Publishers; 3rd Ed., 2004).

[12] J. Goodman, Speckle Phenomena in Optics, (Roberts and Company Publishers; 2010).

[13] G. J. Gbur, Mathematical Methods for Optical Physics and Engineering (Cambridge University Press 2011).

[14] L. D. Landau, E. M. Lifshitz and L. P. Pitaevskii, Electrodynamics of Continuous Media, § 95 (2nd Ed., Reed Educational and Professional Publishing). 